\title{
Gastos públicos direcionados ao combate da covid-19 em Alagoas
}

\section{Public expenditures directed to combat covid-19 in the state of Alagoas}

\author{
Carlos Rodrigo dos Santos ${ }^{1}$
}

Thaynara Maria Pontes Bulhões ${ }^{3}$

Orcid: https://orcid.org/0000-0003-2398-817

Caroline Magna de Oliveira Costa ${ }^{5}$

Orcid: https://orcid.org/0000-0003-4096-4093

\author{
Diane Fernandes dos Santos ${ }^{2}$ \\ Orcid: https://orcid.org/0000-0002-5472-4122
}

Jayane Omena Magna de Oliveira ${ }^{4}$

Orcid: https://orcid.org/0000-0003-2830-9379

Christefany Régia Braz Costa ${ }^{6}$

Orcid: https://orcid.org/0000-0001-6124-8243

\begin{abstract}
Resumo
Introdução: A pandemia da COVID-19 tem apresentado relevância epidemiológica no sistema público de saúde do estado de Alagoas, Brasil. Entretanto, existem poucas publicações científicas sobre o impacto econômico da patologia. Objetivo: Descrever o direcionamento de recursos públicos empenhados no combate à COVID-19 no estado de Alagoas. Métodos: Trata-se de uma pesquisa descritiva com abordagem quantitativa dos dados. Foram incluídas despesas relacionadas a cestas básicas, comunicação, testes para a COVID-19, equipamentos hospitalares, ambulâncias, equipamentos de proteção individual, material médico hospitalar, contratação de obras e serviços de engenharia. A exploração ocorreu no portal da transparência do estado de Alagoas durante o mês de setembro de 2020, a partir dos valores empenhados. O período investigado se deu até o dia 05 de setembro de 2020. Resultados: Constatou-se que Alagoas empenhou o total de R $\$ 39.304 .771,41$ na compra de Equipamentos de Proteção Individual (EPI), material médico-hospitalar, testes para COVID-19 e equipamentos Hospitalares. Além disso, o estado empenhou maiores recursos em assistência hospitalar e ambulatorial com $\mathrm{R} \$ 137.706 .530,81$ e os menores na formação de recurso humanos com R\$ 11.610,90. Destacando-se, o empenho de R\$ 1.008.047, 93 para a vigilância epidemiológica. Conclusões: O estado viabilizou recursos principalmente para elementos que estavam diretamente relacionados à assistência à saúde no enfrentamento da problemática.
\end{abstract}

Palavras-chave: saúde pública; gastos em saúde; infecções por coronavírus

\begin{abstract}
\footnotetext{
${ }^{1}$ E-mail: carlos.rodrigo@souunit.com.br

${ }^{2}$ Universidade Federal de Alagoas. E-mail: dianefernandes@outlook.com.br

${ }^{3}$ Universidade Federal de Alagoas. E-mail: thaybulhoes@gmail.com

${ }^{4}$ Universidade Federal de Alagoas .E-mail: jayaneomena@gmail.com

${ }^{5}$ Universidade Federal de Alagoas. E-mail: carolmagnacosta@gmail.com

${ }^{6}$ Universidade de São Paulo - USP. E-mail: christefany.costa@usp.br
}

Introduction: The COVID-19 pandemic has shown epidemiological relevance in the public health system in the state of Alagoas, Brazil. However, there are few scientific publications on the economic impact of pathology. Objective: To analyze the targeting of public resources committed to combat COVID-19 in the state of Alagoas. Methods: This is a descriptive study, with transversal analysis and quantitative focus of the data. Expenses related to basic food baskets, communication, tests for COVID-19, hospital equipment, ambulances, personal protective equipment, hospital medical supplies, contracting works and engineering services were included. The exploration took place in the transparency portal of the state of Alagoas 
during the month of September 2020, based on the amounts committed. The investigated period was until September 5, 2020. Results: It was found that Alagoas invested a total of R\$ 39,304,771.41 in the purchase of Personal Protective Equipment (PPE), medical and hospital supplies, tests for COVID-19 and Hospital equipment. In addition, the state invested the largest resources in hospital and outpatient assistance with $\mathrm{R} \$ 137,706,530.81$ and the lowest in human resource training with $\mathrm{R} \$ 11,610.90$. Noteworthy, the commitment of $\mathrm{R} \$ 1,008,047,93$ for epidemiological surveillance. Conclusions: The state made resources available mainly for elements that were directly related to health care in facing the problem.

Keywords: public health; health expenditures; coronavirus infections

\section{Introdução}

Ao longo dos anos, o panorama da saúde pública é delineado por inúmeras intervenções econômicas, sociais e políticas, a fim de desenvolver estratégias públicas e assegurar a universalização da saúde ofertada pelo Estado. A partir da reforma sanitária brasileira a saúde tornouse requerida como um direito refletido nas condições de trabalho e qualidade de vida ${ }^{1}$. Assim, esse movimento sanitarista inicial enumerou requisitos imprescindíveis para garantir a democratização da saúde.

Diante da tensão das mobilizações sociais, foi instituído o Sistema Único de Saúde (SUS), que tinha como proposta ofertar a saúde de forma integral. Esse programa foi regulamentado através da lei $\mathrm{n}^{\circ} 8.080 / 1990$. A proposta atribuía aos recursos dos Municípios, Estados e União, além de fontes complementares de financiamento, a oferta da assistência à saúde nos diferentes níveis de atenção ${ }^{2}$. A partir da descentralização da prestação dos serviços de saúde, tornou-se possível ofertar a assistência com maior qualidade e garantir o controle e a fiscalização por parte dos cidadãos.

Assim, os serviços disponibilizados pelo Estado precisam ser estruturados em níveis crescentes de complexidade, incluídos em uma determinada área espacial, organizados diante de variáveis epidemiológicas, e com definição e conhecimento da população a ser atendida ${ }^{3}$. Contudo, esse investimento na saúde pública necessita de recursos, que é refletido em impostos e que por sua vez necessitam ser bem administrados e relatados de forma transparente para a população.

Em face da descoberta do alto potencial de propagação do SARS-CoV-2, novo coronavírus responsável pelo desenvolvimento da patologia reconhecida como COVID-19, despertou-se uma preocupação mundial. Até 18 de outubro de 2020 foram registrados 39.596 .858 casos da COVID-19 e 1.107.374 mortes no mundo. Nesse mesmo intervalo, o Brasil notificou 5.200.300 casos confirmados com 153.214 óbitos $^{4}$. Esse panorama foi tomado como base para visualização do avanço exponencial da transmissão comunitária, definindo-a como relevante problema de saúde pública ${ }^{5}$. A partir da avaliação do alto potencial de infecção do vírus, das repercussões clínicas e da letalidade, a problemática se tornou prioridade para a gestão do estado. Além da preservação da saúde da população, o temor dos gestores esteve/está relacionado ao colapso do sistema de saúde, sobretudo ao direcionamento racional e eficaz de recursos financeiros para a profilaxia e tratamento de pessoas com COVID-19.

Em vista disso, o presente estudo objetiva descrever o direcionamento de recursos públicos para o combate da COVID-19 no estado de Alagoas, Brasil.

\section{Materiais e Métodos}

\section{Amostra e tipo de estudo}

Trata-se de uma pesquisa descritiva com abordagem quantitativa, acerca dos gastos públicos no combate à COVID-19 no estado de Alagoas. 


\section{Delineamento da pesquisa}

A busca ocorreu no portal da transparência do estado de Alagoas durante o mês de setembro de 2020, a partir dos valores empenhados, isso é, dos valores que o governo reserva para efetuar o pagamento de um respectivo bem/serviço. O período investigado se deu entre o mês de março e 05 de setembro de 2020 .

\section{Critérios de Inclusão e Exclusão}

A análise adotou como critérios de inclusão: despesas relacionadas a cestas básicas, comunicação, testes para a COVID-19, equipamentos hospitalares, ambulâncias, equipamentos de proteção individual, material médico hospitalar, contratação de obras e serviços de engenharia, que estão direcionados à promoção e manutenção da saúde do estado de Alagoas. Como critério de exclusão foi descartado qualquer despesa que não fosse direcionada ao gasto da COVID-19 no estado.

\section{Procedimentos}

Os resultados encontrados foram registrados e analisados através de planilhas do Microsoft Excel ${ }^{\circledR}$ para melhor análise e comparação das informações coletadas. Este estudo dispensou a submissão ao Comitê de Ética por se tratar de dados de domínio público, de acordo com a Resolução 466/12 do Conselho Nacional de Saúde.

\section{Resultados}

Os resultados encontrados até a data de elaboração deste estudo demonstram que o estado de Alagoas empenhou o total de $\mathrm{R} \$$ 39.304.771,41 (trinta e nove milhões, trezentos e quatro mil, setecentos e setenta e sete reais e quarenta e um centavos) na compra de Equipamentos de Proteção Individual (EPI), material médicohospitalar, testes para COVID-19 e equipamentos Hospitalares. Por outro lado, o valor empenhado na compra de respiradores foi de $\mathrm{R} \$ 10.513 .800,00$ (dez milhões, quinhentos e treze mil e oitocentos reais $)^{5}$. Os valores dos demais elementos de despesa se encontram detalhados na tabela 1.

Tabela 1: Valores empenhados na compra de materiais para combate da COVID-19 no estado de Alagoas.

Elemento de despesa
Elemento Contábil

Valor empenhado

\begin{tabular}{|c|c|c|}
\hline Cestas Básicas & Material de Distribuição Gratuita & $\mathrm{R} \$ 13.434 .998,4$ \\
\hline Comunicação + Testes para COVID-19 & $\begin{array}{c}\text { Outros serviços de Terceiros/ Pessoa } \\
\text { Jurídica }\end{array}$ & $\mathrm{R} \$ 27.181 .535,85$ \\
\hline Equipamentos hospitalares + Ambulâncias & Equipamentos e material permanente & $\mathrm{R} \$ 11.732 .664,59$ \\
\hline Respiradores & $\begin{array}{l}\text { Rateio pela participação em consórcio } \\
\text { público }\end{array}$ & $\mathrm{R} \$ 10.513 .800,00^{*}$ \\
\hline $\begin{array}{l}\text { Equipamentos de Proteção Individual + } \\
\text { Material Médico-hospitalar + Testes para } \\
\text { COVID-19 + Equipamentos Hospitalares }\end{array}$ & Material de Consumo & $\mathrm{R} \$ 39.304 .771,41$ \\
\hline Plantões Extras & $\begin{array}{c}\text { Outros serviços de Terceiros/ Pessoa } \\
\text { Física }\end{array}$ & $\mathrm{R} \$ 52.331 .769,28$ \\
\hline $\begin{array}{c}\text { Contratação de obras e Serviços de } \\
\text { Engenharia }\end{array}$ & Obras e Instalações & $\mathrm{R} \$ 14.157 .227,92$ \\
\hline
\end{tabular}




\begin{tabular}{cccc}
\hline Outros & - & $\mathrm{R} \$ 28.109 .659,86$ \\
\hline Total & - & $\mathrm{R} \$ 186.252 .627,31$
\end{tabular}

Legenda: * a dotação orçamentária inicial destinada à compra de respiradores pelo Governo do estado de Alagoas foi de R \$ 10.513.800,00 reais. No entanto, após o cancelamento da compra, nenhum investimento com relação a essas máquinas foi de fato realizado.

Além disso, observou-se que no que tange a funções e subfunções das despesas, Alagoas empenhou maiores recursos em assistência hospitalar e ambulatorial com R\$ 137.706.530,81 (cento e trinta e sete milhões, setecentos e seis mil, quinhentos e trinta reais e oitenta e um centavos) e os menores na formação de recursos humanos com R \$11.610,90 (onze mil, seiscentos e dez reais e noventa centavos). Outro item relevante a ser citado é a vigilância epidemiológica, na qual foi empenhada um valor de R\$1.008.047, 93 (um milhão, oito mil, quarenta e sete reais e noventa e três centavos)s, conforme mostra o gráfico 1.

Gráfico 1: Recursos empenhados nas funções e subfunções das despesas para combate da COVID-19 no estado de Alagoas.

\begin{tabular}{|c|c|c|c|}
\hline \multirow{2}{*}{$\begin{array}{l}\text { Assistência hospitalar e ambulatorial } \\
\text { Suporte profilático e terapêutico }\end{array}$} & \multicolumn{3}{|c|}{\begin{tabular}{|r|r|}
$\mid$ & $137.706 .530,81$
\end{tabular}} \\
\hline & \begin{tabular}{|l}
$16.337 .977,76$ \\
\end{tabular} & & \\
\hline Assistência comunitária & $13.351149,9$ & & \\
\hline Educação básica & $8.757 .631,03$ & & \\
\hline Comunicação social & $4.772 .684,23$ & & \\
\hline Administração geral & $3.537 .921,23$ & & \\
\hline Vigilância epidemiológica & $1.088 .047,93$ & & \\
\hline Custodia e reintegração social & $393.575,5$ & & \\
\hline Difusão cultural & 196.800 & & \\
\hline Ensino Superior & 51.355 & & \\
\hline Policiamento & 35.234 & & \\
\hline Defesa Agropecuária & 13.234 & & \\
\hline Formação de recursos humanos & $11.610,9$ & & \\
\hline & $50.000 .000,00$ & $100.000 .000,00$ & $150.000 .000,00$ \\
\hline
\end{tabular}

\section{Discussão}

Em cenário nacional, segundo $\mathrm{o}$ portal de transparência federal até a data da última atualização (25/03), foram empenhados cerca de R $\$ 2,01$ bilhões, destacando-se as transferências de R $\$ 424$ milhões dos fundos estaduais com recursos liquidados e pagos, e de $\mathrm{R} \$ 1,21$ bilhão aos fundos municipais de saúde direcionados ao combate da COVID-19. Além disso, o Governo Federal presume a destinação de cerca de $\mathrm{R} \$ 4,84$ bilhões ao programa de
"Atenção Especializada à Saúde" por parte do Ministério da Saúde 6 .

Ao analisar os valores empenhados na compra de materiais para o combate da COVID-19 no estado de Alagoas, identificou-se um gasto total de R\$ 39.304.771,41 em quatro itens de material de consumo ${ }^{5}$. Em contrapartida, o estado do Ceará gastou $\mathrm{R} \$ 140.374 .771,35$ em apenas um item, que seria material hospitalar ${ }^{7}$. Isso pode ser justificado pela quantidade de pessoas com diagnóstico confirmado para o 
vírus no estado, sendo 80.462 até o dia 5 de setembro de 2020 para Alagoas ${ }^{8}$ e 221.953 até o dia 5 de agosto de 2020 para o Ceará 9 .

Todavia, em relação aos respiradores, Alagoas empenhou um total de $\mathrm{R} \$ 10.513 .800,00$ reais, sendo pagos 4,4 milhões de reais para a compra dessas máquinas. No entanto, a compra foi cancelada, conforme indica o Portal da Transparência do Estado de Alagoas ${ }^{5}$

Sobre isso, a Secretaria Estadual de Saúde (SESAU), esclareceu que já havia realizado o pagamento dos respiradores, mas que a empresa contratada descumpriu os termos do contrato firmado e deixou de efetuar a entrega dos bens, razão pela qual tramita na Justiça, uma ação para reaver os valores que foram pagos ${ }^{10}$.

A COVID-19 pode se apresentar através de uma Síndrome Respiratória Aguda Grave (SRAG), caracterizada por um quadro sintomático crítico que requer internações e/ou cuidados intensivos ${ }^{11}$. Por isso, esse maior investimento em recursos de assistência hospitalar e ambulatorial, devido à necessidade de adquirir equipamentos e insumos para a assistência e disponibilizar leitos por meio da construção e ampliação de unidades hospitalares para atendimento de casos graves $^{12}$

Dentre os itens que tiveram os menores valores empenhados, encontra-se o gasto em vigilância epidemiológica. A partir do conceito de que a vigilância epidemiológica consiste no conjunto de ações que proporcionam o conhecimento, a detecção ou prevenção de qualquer mudança nos fatores determinantes $\mathrm{e}$ condicionantes de saúde individual ou coletiva $^{11}$, o baixo direcionamento de recursos poderá inviabilizar orientações e adoções de decretos de prevenção e monitoramento da dinâmica de disseminação do novo coronavírus. Assim, a falha na monitorização epidemiológica com testes insuficientes para sintomáticos pode ser refletida na subnotificação de casos, o que dificulta a elaboração de estratégias para o controle da doença ${ }^{12}$.
Além disso, a aplicação financeira reduzida em recursos humanos no contexto da pandemia de COVID-19 pode impactar na área da saúde, uma vez que, uma menor qualificação profissional, prejudica o andamento da assistência em saúde, visto que a situação emergencial demanda um maior quantitativo de profissionais capacitados para o cuidado ${ }^{13}$. Todavia, vale destacar que esses valores podem ser suficientes para o estado, porém quando analisados com as demais variáveis, teve um dos menores valores empenhados.

Percebe-se que recursos também foram empenhados para as áreas da educação, comunicação, defesa agropecuária, entre outros. Isso pode ser justificado porque a informação é um instrumento essencial para o alcance do conhecimento da população, permeando sobre os inúmeros âmbitos da educação, sejam eles no contexto político, social ou da saúde. Nesse sentido, o surgimento do SARS-COV-2, ao gerar uma pandemia global, tornou a comunicação ainda mais fundamental. Houve um aumento exponencial pela busca de informações, razão pela qual, em virtude das fontes não confiáveis, acabaram provocando o aumento massivo da disseminação de fake News. Em resposta a essa problemática, o governo tem investido em sites, portais que abordem a COVID-19 de forma confiável e com base em evidências científicas, que buscam levar conhecimento de maneira verdadeira $^{14,15}$.

Já no que tange aos valores empenhados na agropecuária, estes foram investidos em virtude do isolamento social obrigatório, em que foi verificada uma maior procura por alimentos, uma vez que alimentação domiciliar teve um aumento, ocasionando um menor impacto na economia quando comparado com a alimentação externa ${ }^{16}$.

Diante do exposto, a avaliação dos recursos econômicos está cada vez mais presente nos sistemas e serviços de saúde. Conhecer e analisar o destino dos gastos nesse âmbito torna-se relevante, uma vez 
que a divulgação, visibilidade e sistematização dessas informações auxiliam na tomada de decisões e colaboram para reflexão sobre uma "ideal" alocação de recursos disponíveis. Além disso, vale destacar que o conhecimento dos recursos orçamentários não cabe só aos gestores, profissionais de saúde e fornecedores, mas também à população em geral, já que se beneficiam diretamente dos bens e serviços ofertados $^{17-19}$.

\section{Conclusão}

Em face do exposto, foi possível descrever o direcionamento de recursos públicos para o combate da COVID-19 no estado de Alagoas, Brasil. Ao dispor a importância de $\mathrm{R} \$ 39.304 .771,41$ para a compra de EPI, material médico-hospitalar, testes para COVID-19 e equipamentos Hospitalares, o estado viabilizou recursos, principalmente para elementos que estavam diretamente relacionados à assistência à saúde no enfrentamento da problemática. Esse direcionamento de recursos dos cofres públicos é justificado de acordo com as variáveis da qualidade de saúde, pois, influenciam o Estado a desenvolver estratégias governamentais eficazes sem desperdício de recursos, tempo e, sobretudo, de vidas.

Logo, ambiciona-se executar estratégias que minimizem, de alguma forma, a disseminação comunitária da COVID-19, por meio dos investimentos transparentes em saúde, sobretudo no que se relaciona à gestão dos serviços. Sendo assim, é possível fiscalizar o direcionamento de recursos públicos, atribuindo também à população a oportunidade de contribuir e se conscientizar sobre o uso racional de investimentos.

\section{Referências}

1. Guizardi FL, Cavalcanti FO. A gestão em saúde: nexos entre o cotidiano institucional e a participação política no SUS. Interface (Botucatu). 2010 Set;14(34):633-646. Available from: http://www.scielo.br/scielo.php?script=sci_arttext\&pid=S141432832010000300013\&lng=en. https://doi.org/10.1590/S1414-32832010005000013.

2. Brasil. Lei $n^{\circ} 8.080$, de 19 de setembro de 1990. Lei Orgânica da Saúde. Dispõe sobre as condições para a promoção, proteção e recuperação da saúde, a organização e o funcionamento dos serviços correspondentes e dá outras providências. Diário Oficial da União. 1990 Set. 18.

3. Dias LNS, Matias-pereira J, Farias MRS, Pamplona VMS. Fatores associados ao desperdício de recursos da saúde repassados pela união aos municípios auditados pela Controladoria Geral da União. Rev. contab. finanç. 2013;24(63):206-218. [Cited 2020 Oct. $18]$.

Available

from: http://www.scielo.br/scielo.php?script=sci arttext\&pid=S151970772013000300004\&lng $=$ en\&nrm=iso.

4. OMS. Painel de controle da doença coronavírus da OMS (COVID-19) [Internet]. [cited 2020 Oct. 18] Available from: https://covid19.who.int/.

5. Alagoas. Estado de Alagoas. Controladoria Geral do Estado. Portal de transparência Graciliano Ramos - Alagoas [Internet]. [cited 2020 sept 05]. Available from: http://transparencia.al.gov.br/despesa/covid19/.

6. Brasil. Controladoria Geral da União. Portal da transparência [Internet]. [cited 2020 sept 05]. Available from: http://www.portaltransparencia.gov.br/comunicados/603503portal-da-transparencia-divulga-gastos-federais-especificos-para-combate-aocoronavirus.

7. Ceará. Governo do Estado do Ceará. Portal da transparência. Recursos aplicados no combate ao coronavírus [internet]. [cited 2020 sept 08]. Available from: https://cearatransparente.ce.gov.br/portalda-transparencia/paginas/coronavirus-despesas. 
8. Alagoas. Estado de Alagoas. Secretaria Estadual de Saúde de Alagoas. Centro de Informações Estratégicas e Resposta em Vigilância em Saúde CIEVS/AL. n. 183 [Internet]. 2020 [cited 2020 sept 08]. Available from: https:/www.saude.al.gov.br/wpcontent/uploads/2020/09/Informe-Epidemiologico-COVID-19-no-183-05-9-2020-1.pdf.

9. Ceará. Governo do Estado do Ceará. Boletim epidemiológico: Doença pelo coronavírus (COVID-19). Secretaria Executiva de Vigilância e Regulação Em Saúde - SEVIR. [Internet]. 2020B [cited 2020 sept 08]. Available from: https://coronavirus.ceara.gov.br/boletins/.

10. Alagoas. Estado de Alagoas. Secretaria Estadual de Saúde de Alagoas. AL aciona Justiça para reaver R \$ 4,4 milhões repassados para aquisição de respiradores [Internet]. 2020 [cited 2020 sept 08]. Available from: https://www.saude.al.gov.br/al-aciona-justica-para-reaverr-44-milhoe s-repassados-para-aquisicao-de-respiradores/.

11. Ministério da Saúde; Secretaria de Vigilância em Saúde. Departamento de Vigilância Epidemiológica. Guia de Vigilância Epidemiológica. Brasília. 2009.

12. Hill B. The COVID-19 pandemic. Br J Nurs [Internet] 2020 Apr [cited 2020 September 07]; 29(8):456. Available from: https://www.magonlinelibrary.com/doi/full/10.12968/bjon.2020.29.8.456.

13. Oliveira WK, Duarte E, França GVA, Garcia, LP. Como o Brasil pode deter a COVID-19. Epidemiol. Serv. Saude [Internet]. 2020. [cited 2020 September 07]; Brasília, 29(2):e2020044. Available https://www.scielosp.org/pdf/ress/2020.v29n2/e2020044/pt.

14. Barreto ML, Barros AJD, Carvalho MS, Codeço CT, Hallal PRC, Medronho RA, et al. O que é urgente e necessário para subsidiar as políticas de enfrentamento da pandemia de COVID-19 no Brasil? Rev Bras Epidemiol [Internet]. 2020 [cited 2020 September 07]; 23: e200032. Available from: http://www.scielo.br/scielo.php?script=sci_arttext\&pid=S1415790X2020000100101\&lng=en.EpubApr22,2020 http://dx.doi.org/10.1590/1980549720200032.

15. Sousa Júnior, JH, Raasch M, Soares JC, Ribeiro LVHAS. Da Desinformação ao Caos: uma análise das Fake News frente à pandemia do Coronavírus (COVID-19) no Brasil. Cadernos de Prospecção [Internet]. 2020 [cited 2020 October 19]; 13(2), Edição Especial. Available from: https://cienciasmedicasbiologicas.ufba.br/index.php/nit/article/view/35978.

16. Letouze P, Souza Júnior JIM, Castelo Everton BL, Barbosa GV. COMUNICAÇÃO DE GOVERNO NA INTERNET SOBRE O COVID-19: um benchmarking descritivo. Revista Observatório [Internet]. 2020 [cited 2020 October 19]; 6(3)Especial 1. Available from: https://sistemas.uft.edu.br/periodicos/index.php/observatorio/article/view/9493.

17. Martha Júnior GB. Uma agropecuária forte amortece os impactos da Covid-19. Revista de Política Agrícola [Internet]. 2020 [cited 2020 October 19]; 1(2). Available from: https://seer.sede.embrapa.br/index.php/RPA/article/view/1612.

18. Silva MT, Silva EN, Pereira MG. Análise de impacto orçamentário. Epidemiol. Serv. Saúde. [Internet]. 2017 [cited 2020 October 19]; 26(2):421-424. Available from: https://www.scielo.br/pdf/ress/v26n2/2237-9622-ress-26-02-00421.pdf.

19. Silva EN, Silva MT, Pereira MG. Estudos de avaliação econômica em saúde: definição e aplicabilidade aos sistemas e serviços de saúde. Epidemiol. Serv. Saúde. [Internet]. 2016 [cited 2020 October 19]; Brasília, 25(1):205-207. Available from: http://scielo.iec.gov.br/scielo.php?script=sci_arttext\&pid=S1679-49742016000100023.

Como citar este editorial:

Santos CR, Santos DF, Bulhões TMP, Oliveira JOM, Costa CMO, Costa CRB. Gastos públicos direcionados ao combate da covid-19 em Alagoas. Rev. Aten. Saúde. 2021; 19(67): 26-32. 